\title{
EXPERIMENTS AT THE VIRTUAL NATIONAL LABORATORY FOR HEAVY ION FUSION
}

\author{
P. A. Seidl, F.M. Bieniosek, C.M. Celata, A. Faltens, J.W. Kwan, S.A. MacLaren, \\ D. Ponce, D. Shuman and S. Yu \\ Lawrence Berkeley National Laboratory, Berkeley, CA, USA \\ L. Ahle, S. Lund, A. Molvik and T.C. Sangster \\ Lawrence Livermore National Laboratory, Livermore, CA, USA
}

\section{Abstract:}

An overview of experiments is presented, in which the physical dimensions, emittance and perveance are scaled to explore driver-relevant beam dynamics. Among these are beam merging, focusing to a small spot, and bending and recirculating beams. The Virtual National Laboratory for Heavy Ion Fusion (VNL) is also developing two driver-scale beam experiments involving heavy-ion beams with $\mathrm{I}_{\text {beam }} \sim 1$ Ampere to provide guidance for the design of an Integrated Research Experiment (IRE) for driver system studies within the next 5 years. Multiple-beam sources and injectors are being designed and a one-beam module will be built and tested. Another experimental effort will be the transport of such a beam through $\sim 100$ magnetic quadrupoles. The experiment will determine transport limits at high aperture fill factors, beam halo formation, and the influence on beam properties of secondary electron production and neutral atom desorption from the vacuum pipe wall. Research into driver technology will be briefly presented, including the development of ferromagnetic core materials, induction core pulsers, multiple-beam quadrupole arrays and plasma channel formation experiments for pinched transport in reactor chambers. 


\section{INTRODUCTION}

The USA Virtual National Laboratory for Heavy Ion Fusion program is completing a sequence of low current and low kinetic energy experiments aimed at understanding heavy-ion fusion (HIF) beam dynamics using scaled perveance and emittance. Such experiments are useful for exploring beam transport as a function of the depressed $(\sigma)$ and undepressed $\left(\sigma_{\mathrm{o}}\right)$ phase advances per lattice period, perveance $(\mathrm{K})$, and emittance $\varepsilon$ at a relatively small scale and cost. Three experiments fall in this category: four-to-one beam merging, recirculation/bending and scaled beam focusing. The phase space evolution due to the beam manipulations in these experiments is the primary experimental result. These scaled experiments address beam dynamics relevant to HIF but miss effects of the high space charge potential and focusing gradients.

The Integrated research experiment (IRE) will be the unifying motivation for the program for the next few years[1]. The IRE is intended to check driver scale beam dynamics and systems integration to enable confident driver design. In the IRE, the current per beam will be similar to that in a driver, but the final kinetic energy will be $\sim 1 / 10$ that of a driver, mainly for economic reasons. Beam quality, characterized by the six-dimensional phase space density, should be similar to a driver (normalized emittance $\varepsilon_{\mathrm{n}}<5 \mathrm{~mm}$-mrad, momentum spread $\Delta \mathrm{p} / \mathrm{p}<10^{-2}$, pulse duration $\Delta \mathrm{t}=5 \mathrm{~ns}$ after drift compression at $\sim 200 \mathrm{MeV}$ ). For more on the theoretical and computational work aimed at the IRE, see ref. [2].

Experiments with IRE-scale beams will explore injection and transport with high space charge potential to test issues not addressed with the scaled experiments. Other 
experiments will test hardware needed for the IRE. Four IRE-related experimental efforts are described here: The High Current Experiment (HCX), magnetic quadrupole array development, induction core development, and channel transport. Ion source and injector development is discussed elsewhere in these proceedings [3,4]. A principal objective of the injector experimental program is to develop a working injector module suitable for the multiple beam configuration of the IRE. This ion source and injector development, and the HCX, will be the main beam experimental efforts for the next few years.

\section{SCALED MERGING}

In several induction linac driver designs, electrostatic quadrupole arrays are the preferred focusing elements at low kinetic energy where the lattice period is short $(\mathrm{L}<1$ m) and the quadrupole occupancy should be as high as possible. At higher kinetic energy, superconducting magnetic quadrupole arrays are advantageous due to their greater focusing strength and the relaxed lattice spacing required to transport the beams. To date, cost optimization of accelerator design studies point to larger apertures (and greater beam current per beam) for magnetic quadrupoles than for electrostatic quadrupoles. Thus merging groups of beams from the electrostatic part of the driver into fewer magnetic quadrupole transport channels is of interest, provided the emittance growth in the merging process is limited to prevent the loss of focusability on the fusion target. In the merging process there are significant contributions to the emittance growth from both single particle dynamics and nonlinear space charge forces.

There is a significant emittance budget (a factor $\sim 10^{3}$ ), as measured by the 6-D phase space of the beams near the (contact ionization) sources compared to the emittance 
required to focus the beams on the converter of an indirect drive fusion target. There will be some inevitable emittance growth in the transport of the beams through thousands of quadrupoles (e.g., due to nonlinear fields from focusing imperfections and image charges and currents) and also from advantageous beam manipulations such as merging, bending and drift compression.

At LBNL, a 4:1 beam merging experiment has been completed. The combining apparatus is designed to pack the beams into a configuration shown in Fig. 1, where the beam-edge to beam-edge separation is $\Delta \approx 4 \mathrm{~mm}$. Four $2.7 \mathrm{~mA}\left(\mathrm{Cs}^{+}, 160 \mathrm{keV}\right)$ beams are each focused by four electrostatic quadrupole channels (Q1-Q4), whose axes converge at a $6^{\circ}$ angle and aim the beams into a combined function dipole-quadrupole element (QD5). The dipole field strength is set to remove the $6^{\circ}$-centroid angle and aim each beam into a common downstream AG lattice. The quadrupole gradient settings of Q1QD5 are set to match the circular beams from the source to the desired final elliptical shape (with a zero envelope divergence angle) after QD5. Element QD5 is comprised of 71 tungsten rods $(\approx 10 \mathrm{~cm}$ long) with voltages set on each rod to approximate the desired field structure. A more detailed description of this experiment is given in [5].

Although the individual beams have much lower current than in a driver, due to the low kinetic energy the perveance is driver scale at $\mathrm{K}=3 \times 10^{-4} /$ beam, the normalized emittance is $0.025 \mathrm{~mm}$-mrad and the transport through the $11-12 \mathrm{~mm}$ radius apertures of Q1-Q4 is dominated by space charge. The maximum envelope radius in Q1-Q4 is $\approx 9 \mathrm{~mm}$, occurring in Q3. 
Data from two diagnostic stations (two and 22 quadrupoles after QD5) downstream of the merge point are discussed here. The HIBEAM 2D particle-in-cell code was used to model the experiment and help interpret the results [6].

\section{Results}

Phase space measurements two quadrupoles, $(0.3 \mathrm{~m})$ downstream, of QD5, show that the individual beamlets inside the merged beam are still distinct. The projection of the transverse phase space onto the $\mathrm{x}-\mathrm{x}^{\prime}$ axes results in two beams overlapping near the origin. The distributions resemble PIC simulations though there are some residual misalignments of the individual beams at the few $\mathrm{mm}$ and mrad level. At this point in the lattice the potential energy from the non-linear space charge of the $10.3 \mathrm{~mA}$ merged beam has not yet thermalized and contributed to emittance growth. Beam loss is negligible at this diagnostic.

Twenty-two quadrupoles, (5 m) downstream of QD5, the experimental phase space is characteristic of a single beam with envelope parameters matched to the lattice quadrupole strengths $\left(2.74 \mathrm{kV} / \mathrm{cm}^{2}\right)$. At this point $\approx 2 \lambda_{\mathrm{p}}$ plasma oscillations $\left(\lambda_{\mathrm{p}}=\mathrm{v}_{\mathrm{z}} / \omega_{\mathrm{p}}\right)$ after the merge the distribution appears uniform and characteristics of the merged beamlets have disappeared. This is shown in fig. 2, where the predicted PIC phase space orientation and density of the merged beam matches that of the experiment rather well. The simulations also indicate that the emittance growth has reached a plateau. Quantitatively, the experimentally observed emittance is $0.2 \mathrm{~mm}-\mathrm{mrad}$ for $97.5 \%$ of the integrated signal measured at this diagnostic station. Approximately $\sim 10 \%$ of the beam has already been lost at this point. Roughly $1 / 5$ of this loss can be attributed to beam background gas interactions. The remainder is due to loss of halo ions at large radii 
striking the machine apertures (the electrodes are at $r=27 \mathrm{~mm}$ ), which is compounded by alignment imperfections. Thus the experimentally determined $\varepsilon_{\mathrm{n}}=0.2 \mathrm{~mm}-\mathrm{mrad}$ corresponds to $\approx 88 \%$ of the initially merged beam. In the PIC simulation, the emittance of macroparticles inside the box in Fig. 2 (corresponding to the experimental sensitivity and measurement boundary) is also $0.2 \mathrm{~mm}$-mrad for $88 \%$ of the initially merged beam (5\% of the macroparticles are lost due to scraping upstream; an additional $7 \%$ of halo particles lie outside the box but have not yet struck the machine aperture). Similar results were obtained by comparing the vertical phase space to the PIC simulation.

This level of agreement between the experiment and the simulation will allow us to design full-scale merging systems with confidence. The results indicate that for some HIF driver designs, the phase space dilution from merging is acceptable. The best driver design will depend on the evolution of target design, driver system optimization, and results such as these.

\section{BENDING AND RECIRCULATION}

A recirculating induction accelerator driver would allow the use of considerably fewer induction core modules and pulsers and a lower acceleration gradient, since the modules can be reused as the beam recirculates in a ring. This may lead to a less expensive driver. Compared to the state of the art in synchrotron beam physics, one significant new challenge is the high space charge of the beam. By rapid acceleration relative to synchrotrons it is expected that phase space dilution due to resonance crossing would be mitigated. Compared to the HIF induction linac approach, the recirculator requires a greater beam pulse length, lower vacuum system pressure, higher induction core pulser repetition rate $(\sim 100 \mathrm{khz})$ and rapidly ramping dipole bending magnets. In 
addition to these technological challenges there are physics issues associated with intense beam propagation and control in a ring.

At LLNL, a small recirculator experiment was designed to study some of the beam dynamics questions (insertion and extraction, steering, emittance growth) of this alternate HIF induction accelerator approach [7]. The recirculator was designed to accelerate $2 \mathrm{~mA} \mathrm{~K}{ }^{+}$ions from $80 \mathrm{keV}$ to $320 \mathrm{keV}$ in 15 laps. The corresponding dimensionless perveance is $2-4 \times 10^{-4}$, and the tune depression spans $0.21<\sigma / \sigma_{\mathrm{o}}<0.27$.

In 1999 , construction was stopped after the first $90^{\circ}$-bend section due to consideration of the relative risk of the recirculator approach and the need for consolidation of experimental efforts in the VNL in order to make acceptable progress toward the IRE. Thus some of the significant challenges noted above remain for this HIF driver approach: insertion, extraction, and emittance growth after several laps of acceleration. The experiment did, however, operate as a $90^{\circ}$ bend and has demonstrated coordinated bending and acceleration, good beam centroid control, and provided information about emittance growth of strongly space charge dominated beams in bends.

\section{Results}

The $80 \mathrm{keV} \mathrm{K}^{+}$alumino-silicate source and diode are followed by an electrostatic matching section before insertion into the bend section. Quadrupole focusing in the bend section is achieved with permanent magnets $\left(\mathrm{B}_{\max } \approx 0.3 \mathrm{~T}, \mathrm{r}_{\mathrm{pipe}}=3 \mathrm{~cm}\right)$ and the dipoles are ramped electrostatic elements following each quadrupole. Four induction acceleration modules add $2.0 \mathrm{keV}$ kinetic energy through the bend section, corresponding to a $\sim 10 \%$ kinetic energy increase per lap. Capacitively coupled beam diagnostics were used to measure the beam centroid. Parallel slit scanners and a gated beam imaging diagnostic 
measured the phase space. Phase space measurements at the end of the bend section (for a coasting beam) show some distortions due to nonlinearities, and the emittance $\left(\varepsilon_{\mathrm{nx}} \approx\right.$ $0.047 \mathrm{~mm}-\mathrm{mrad}, \varepsilon_{\mathrm{ny}} \approx 0.069 \mathrm{~mm}-\mathrm{mrad}$ ) is somewhat larger than predicted by PIC simulations $\left(\varepsilon_{\mathrm{nx}(\mathrm{PIC})} \approx \varepsilon_{\mathrm{ny}(\mathrm{PIC})}=0.04 \mathrm{~mm}-\mathrm{mrad}\right)$. PIC simulations through multiple laps predict that most of the emittance growth should occur in the first of the 15 laps. Transport of the beam through at least a few laps would be needed to resolve the discrepancy between the PIC and the data. Acceleration of the beam through the induction gaps gave beam centroid measurements within $2 \mathrm{~mm}$ of the central orbit, sufficient for further stable transport (see fig. 3). These and other experimental results are presented in detail in ref. [8].

\section{SCALED FOCUSING}

The Scaled Final Focus Experiment at Berkeley Laboratory is based on the HIBALL-II final focus subsystem design [9]; both the design and experiment consist of a set of six magnetic quadrupoles to ballistically focus an ion beam to a small spot. By properly scaling the physics parameters that relate particle energy and mass, beam current, beam emittance, and focusing field, we replicate the dynamics of a full driver beam in a small laboratory beam $\left(K=2.2 \times 10^{-5}\right)$. The $1 / 10$ scale pulsed electromagnet conductor design accurately represents a full scale focusing magnet; two layers of coils are arranged to approximate the desired $\cos (2 \theta)$ current distribution using strategies common to superconducting magnet design. The higher order multipoles (and fringe fields at the magnet ends) have the correct scaled effect on the experimental ion beam since the ion trajectories ( $\mathrm{r}$ and $\mathrm{dr} / \mathrm{dz}$ ) are also 1/10 scale compared to the Hiball-II design. Chromatic effects are studied by detuning the magnet gradients with respect to 
the relatively monoenergetic $(\Delta \mathrm{p} / \mathrm{p}<1 \%)$ and constant-current beam. In a driver, a serious issue is the current variation (due to the last stages of the drift compression) through the final focus elements to create the required beam current pulse at the fusion target. This effect is not reproduced in the experimental results presented to date. See Ref. [10] for a detailed report of the experiment.

The expected emittance-limited (scaled) focal spot for the HIBALL-II design was observed: $\mathrm{r}_{\text {(spot) }} \sim 0.3 \mathrm{~mm}$ for $95 \mu \mathrm{A} \mathrm{Cs}^{+}$(see Fig. 4). We later explored the focusing and neutralization possibilities for higher perveance beams $\left(400 \mu \mathrm{A}, \mathrm{K}=9 \times 10^{-5}\right)$. A thin, hot filament placed after the last focusing magnet emits copious low-energy electrons. By charge neutralization, we have transformed a space charge dominated spot $\left(\mathrm{r}_{(\text {spot })}=3.0 \mathrm{~mm}\right)$ to a significantly smaller emittance limited focal $\operatorname{spot}\left(\mathrm{r}_{(\mathrm{spot})} \sim 1.1 \mathrm{~mm}\right)$. It appears that the degree of neutralization is $\approx 80 \%$. This experimental work begins to address a focusing approach appropriate for high perveance beams, which would permit fewer beams with higher perveance and identical total charge to achieve the same fusion target gain. High perveance is also of interest because lower kinetic energy beams allow higher gain fusion targets [11]. The experiment will also benchmark our PIC simulations of chamber focusing systems [12].

\section{ION SOURCE AND INJECTOR DEVELOPMENT}

We will use the existing $2 \mathrm{MV}$ injector for the high current experiment. It consists of a 17-cm diameter contact ionization source, a cylindrically symmetric diode section ( 0 $<\mathrm{V}<750 \mathrm{kV}$ ) followed by an electrostatic quadrupole (ESQ) acceleration section that is biased to accelerate the beam while providing transverse focusing $(750 \mathrm{kV}<\mathrm{V}<2 \mathrm{MV})$. The beam profile was found to be hollow at the injector exit, and this undesirable non- 
uniformity has been the topic of experimental investigation for the past year. Most of the recent measurements have diagnosed the beam after the diode section, and before the ESQ. See Ref. [3] for a thorough description of the experiment.

The first round of measurements with the $2 \mathrm{MV}$ injector used an alumino-silicate contact-ionization $\mathrm{K}^{+}$source, but more recent measurements used a contact-ionization source where Cs atoms adhere to a bare, porous tungsten substrate. The two types of sources qualitatively give the same (non-uniform) beam profile. Thus, it is doubtful that the choice of ion emitter technology is responsible for the hollow beam.

At the exit of the diode, the beam profile exhibited a strong central peak $(\approx 2 \mathrm{~mm}$ FWHM) in addition to an enhanced current density near the edge of the beam. After

considerable improvements to the vacuum system pressure near the source (now $\sim 1 \times 10^{-6}$ Torr) the amplitude of the central peak has been reduced substantially. EGUN simulations qualitatively reproduce the persistent experimental feature of enhanced current density at the edge of the beam. Therefore, the non-uniformity is mainly due to edge aberrations in the diode optics, and it appears that it can be corrected by adjustments to the geometry. In particular, reducing the transverse dimensions of the source (increasing the source emission current density to maintain the same total current) should diminish the aberrations to an acceptable level, and produce a beam suitable for use in planned high-current transport experiments.

\section{HIGH CURRENT EXPERIMENT (HCX)}

Earlier HIF quadrupole transport experiments have had beam currents (or line charge densities) that were an order of magnitude (or more) smaller than those needed in a full-scale driver. The HCX will be the first transport experiment with driver-scale line 
charge density and pulse duration. It will address important issues related to the preservation of good beam quality (focusability), beam sensing and alignment, beam size, secondary electron effects and halo production at high space-charge intensity over long pulses. The HCX will explore transport in an IRE relevant regime with a (less costly) single beam without acceleration and will provide a sound basis for the IRE. Of particular importance to HIF (heavy ion fusion) multiple beam induction linear accelerators is the aperture fill factor, $\mathrm{a} / \mathrm{R}$ : the ratio of the maximum beam envelope radius to the beam pipe radius. Fill factors exceeding $50 \%$ are desirable to minimize the focusing array size and in turn the amount of induction core material needed for acceleration.

The HCX will be a single-beam transport experiment using electrostatic and magnetic quadrupoles and a long pulse $(\sim 20 \mu \mathrm{s})$ low energy coasting beam $(1.6-2 \mathrm{MeV}$ $\mathrm{K}^{+}$or $\mathrm{Cs}^{+}$). The HCX will have two phases:

HCX Phase I: will be a relatively short precursor. The goal is to measure the transport of a driver scale beam (>300 mA and $\sim 7 \mu$ s pulse length) through a combination of 15-30 electrostatic and pulsed magnetic quadrupoles for various fill factors. Prototype electrostatic quadrupole arrays with aperture diameter $4.6 \mathrm{~cm}$ and an operating electrode potential of $\pm 60-70 \mathrm{kV}$ were developed for the Elise project, which was designed for a line charge density of $\lambda \approx 0.25 \mu \mathrm{C} / \mathrm{m}$. Fabrication techniques of this design will be adapted to the phase I of the HCX.

HCX Phase II: will use more current and pulse duration (>500 mA, > $15 \mu \mathrm{s})$ with $\geq 100$ quadrupoles in order to be more sensitive to emittance growth and halo formation. Furthermore, we will integrate superconducting magnets into the experiment, in order to 
gain experience with this focusing technology that will be a major part of beam transport in a driver and possibly the IRE. Present HCX lattice designs have a half-period $\mathrm{L}<20 \mathrm{~cm}$, lattice occupancy $\eta \approx 0.5$, the radius to the current carrying superconductors is $\approx 3 \mathrm{~cm}$ and the peak field in the conductors is $3-4 \mathrm{~T}$.

\section{QUADRUPOLE ARRAY DEVELOPMENT}

A prototype IRE room-temperature pulsed magnetic quadrupole array [13] is being fabricated (Fig. 5). A modified $\cos (2 \theta)$ current distribution (due to the presence of neighboring quadrupoles, the desired current distribution is not exactly $\cos (2 \theta))$ is placed inside an elliptical iron boundary. Elliptical apertures follow the beam profile, reducing the pulser requirements and allow higher aperture fill factors. Each aperture has semimajor and minor axes of 5 and $3 \mathrm{~cm}$. The peak field in the coils is $\approx 1.8 \mathrm{~T}$. The design is directly applicable to low-field superconducting arrays and can be adapted for higher fields.

For economic reasons, superconducting magnet arrays will be necessary in a driver. An example of a recent superconducting array design [14] is shown in Fig. 6. It uses a flexible, round mini-cable, which can easily be bent in any direction and which operates at a relatively low current (2-3kA). A NbTi, round mini-cable with 37 strands was developed for this application. Because of its flexibility, the cable facilitates automated, cost-effective coil winding and eliminates the necessity for complex coil end designs. The coils are wound onto a cylindrical mandrel. Since the coils are in close proximity to each other, flux penetrates into neighboring cells, enhancing the field in neighboring cells by more than $30 \%$. 
A quite different design approach is shown in Fig 7. Each quadrupole uses two layers of Rutherford style cable with the coils laid flat on a square support structure. This approach has attractive fabrication features for a multiple beam array [15]. The cable current is $\sim 6 \mathrm{kA}$.

Derived from these two design efforts, two single beam prototype superconducting magnets will be fabricated and tested this year. The gradient and coil lengths will be consistent with the HCX lattice parameters given above.

\section{CORE DEVELOPMENT}

Tests of various induction core materials are shown in Fig. 8. Efficiency of transferring energy to a heavy-ion beam is calculated based on the experimentally determined core losses, which increase with the magnetization rate $\mathrm{dB} / \mathrm{dt}$. As the beam velocity in the induction linac increases, the beam current increases, the pulse length decreases, and the required magnetization rate of the ferromagnetic material increases. The data show that for various core materials, good accelerator efficiencies are possible. The choice of material is ultimately a trade off between price and performance. For more experimental details see Ref. [16].

\section{PINCHED BEAM TRANSPORT}

At the end of a driver, where the beam must be focused longitudinally and transversely to satisfy the fusion target requirements, plasma channel, or Z-pinched beam transport could ease the transverse beam emittance requirements and overcome the strong repulsive space charge forces. The challenge is to create a stable plasma between the reactor chamber wall and the fusion target. The plasma current should be $\sim 50 \mathrm{kA}$ in order to confine the heavy ion beam, and the background gas density requirements for the 
channel formation must be compatible with multiple scattering and energy loss of the beam by the background gas.

The LBNL plasma channel experiment has demonstrated $40 \mathrm{~cm}-$ long stable Zpinches with a peak current of $\approx 55 \mathrm{kA}$. Recent experiments have aimed to characterize the properties of the plasma channel: time dependence of the plasma density spatial distribution, and magnetic field. It has been empirically determined that a laser-initiated plasma pre-pulse stabilizes the formation of the main plasma channel considerably. In the experiment, a 10ns Nd:YAG laser forms an ionization path, followed by a $7 \mathrm{kV}$ discharge. The discharge forms a gas density depression and defines the discharge path for the main pulse $\approx 35 \mu$ s later. For the main pulse the capacitor bank, charged to 15 $30 \mathrm{kV}$, is discharged, forming a plasma channel carrying a peak current of $\geq 30 \mathrm{kA}$. The combination of prepulse and main pulse eliminates premature breakdown to the chamber wall. A Michelson interferometer is used to measure electron density in the main plasma channel. Figure 9 shows some of the data measured vs. time. The peak density is $\approx 3 \times 10^{16} / \mathrm{cm}^{3}$, and is sufficient for charge neutralization of an intense heavy-ion beam. Note that the minimum FWHM of the plasma density occurs at $t \approx 2 \mu \mathrm{s}$, which is a few $\mu \mathrm{s}$ before the peak plasma current. It is expected that a heavier gas will delay the pinch formation so that it coincides with the maximum plasma current and pinch field.

Upcoming experimental effort will be devoted to measuring the pinch field, and the properties of the prepulse plasma. Experimental details are presented in Ref. [17].

\section{SUMMARY}

Scaled experiments (merging, recirculation/bending, scaled focusing) are completed or near completion. The main beam experiments in advance of the IRE will 
be the development of a multiple beam driver-scale injector and the HCX. These experiments, along with technology development, will place the design of the IRE on a firm foundation.

\section{ACKNOWLEDGEMENTS}

This work is supported by the Director, Office of Energy Research, Office of Fusion Energy Science, US DOE, under Contract \# DE-AC03-76SF00098 and W-7405ENG-48.

\section{REFERENCES}

[1] R. Bangerter, "The heavy-ion fusion program in the USA", these proceedings.

[2] A. Friedman, "Beam simulations for IRE and driver - status and strategy", these proceedings.

[3] F. Bieniosek, "Development of a 2MV injector for HIF", these proceedings.

[4] J. Kwan, "Ion sources and injectors for HIF induction linacs", these proceedings. T.C. Sangster et al., "A new 500-kV ion source test stand for HIF”, these proceedings.

[5] P.A. Seidl et al., "Progress on the scaled beam-combining experiment at LBNL”, Nucl. Inst. Meth. A415 (1998) 243.

[6] W. M. Fawley, "Numerical Simulation Studies of the LBNL Heavy- Ion Beam Combiner Experiment”, Proceedings of the 1997 Particle Accelerator Conference, Vancouver, Canada May 1997, IEEE Cat. \# 97CH36167, pg. 1923-1925.

[7] J. J. Barnard et al., "Physics design and scaling of recirculating induction accelerators: from bench-top prototypes to drivers”, Fus. Eng. \& Design, 32-33 (1996) 247. M. A. Newton et al., "Engineering development for a small-scale recirculator 
experiment", Fus. Eng. \& Design, 32-33 (1996) 259. T. C. Sangster et al., "Status of experiments leading to a small recirculator", Nucl. Inst. Meth. A415 (1998) 314.

[8] L. Ahle et al., "Results from the Recirculator Project at LLNL", these proceedings.

[9] "HIBALL-II: An Improved Conceptual Heavy Ion Beam Driven Fusion Reactor Study", KfK 3840, Kernforschungszentrum Karlsruhe (July 1985), pp.57-71.

[10] S. A. Maclaren et al., "Results from the scaled final focus experiment", these proceedings.

[11] D. Calahan-Miller, "Progress in heavy-ion fusion targets", these proceedings.

[12] M. DeHoon et al., "Simulation of the LBNL scaled final focus experiment", these proceedings.

[13] D. Shuman et al., "A pulsed elliptical quadruple array for transport of multiple high current beams" Proceedings of the 1999 Particle Accelerator Conference, ISBN 0-780305576-8 (1999) 3339.

[14] R. Meinke et al., "Development of Quadrupole Arrays for Heavy-Ion Fusion” IEEE Transactions on Applied Superconductivity, Vol. 10, \#1, (2000) 192.

[15] S. Caspi and N. Martovetsky, "The LLNL quadrupole array”, LBNL internal note SC-MAG-676 (1999).

[16] A. W. Molvik, "Induction core efficiency at $5 \mathrm{~Hz}$ ", these proceedings.

[17] D. M. Ponce et al., "Diagnostics of plasma channel for HIF transport", these proceedings. C. Niemann et al., "Development of a faraday polarimeter for magnetic field measurements in a discharge channel for ion beam transport", these proceedings. 


\section{Figure Captions}

Figure 1: $\mathrm{x}-\mathrm{y}$ configuration of beams immediately after passing through QD5.

The hatched area represents the beam envelopes; $\mathrm{a}=6.5 \mathrm{~mm}, \mathrm{~b}=3.7 \mathrm{~mm}, \Delta=4 \mathrm{~mm}$.

Figure 2: Experimental phase space (top) measured 22 quadrupoles downstream of QD5, compared to the PIC simulation. The lower box approximates the region inside which the beam signal was above the background noise level.

Figure 3: Beam centroid position measured through the bend section with and without acceleration. With acceleration, the centroid is within $\pm 2 \mathrm{~mm}$ of the central orbit.

Figure 4: Vertical beam profile of the focal spot measured for a beam current and emittance scaled from the HIBALL-II design.

Figure 5: An IRE prototype 21 beam array is being fabricated and will be tested this year.

Figure 6: A superconducting quadrupole array design based on round mini-cable wound on a cylindrical mandrel.

Figure 7: A superconducting quadrupole array design based on Rutherford cable wound onto a flat support structure.

Figure 8: Acceleration efficiency using three induction core materials.

Figure 9: Plasma channel density from Michelson interferometer data. 